\title{
Complex interrelations between self-reported oral health attitudes and behaviors, the oral health status, and oral health-related quality of life
}

This article was published in the following Dove Press journal:

Patient Preference and Adherence

\author{
Alexandra Lucia Vigu' \\ Dorin Stanciu ${ }^{2}$ \\ Lucia Maria Lotrean ${ }^{3}$ \\ Radu Septimiu Campian ${ }^{4}$ \\ 'Department of Dental Materials \\ and Ergonomics, "Iuliu Hațieganu" \\ University of Medicine and Pharmacy, \\ ${ }^{2}$ Department of Psychology and \\ Pedagogy, Technical University of Cluj- \\ Napoca, ${ }^{3}$ Department of Community \\ Medicine, "Iuliu Hațieganu" \\ University of Medicine and Pharmacy, \\ ${ }^{4}$ Department of Oral Rehabilitation, \\ Health and Management, "Iuliu \\ Hațieganu" University of Medicine and \\ Pharmacy, Cluj-Napoca, Romania
}

Purpose: The purpose of this study was to develop and test a moderated mediation model that was able to describe the relationships between oral health-related attitudes and behaviors, oral health status (OHS), and oral health-related quality of life. The hypothesized relations corresponded to research questions such as "is a person's oral health predicted by the actions that person takes in order to prevent oral health conditions?" and "do individuals with better oral health also have higher levels of oral health-related quality of life?".

Materials and methods: A cross-sectional correlational study with selected predictor variables was conducted in Cluj-Napoca, Romania, among 191 participants, enrolled in the fourth and sixth years of study at the Dentistry School of the Medicine and Pharmacology, University of Cluj-Napoca. Participants completed the Hiroshima University Dental Behavior Inventory (HUDBI) questionnaire targeting specific behavior and attitude with respect to their dental self-care, Oral Health Impact Profile (OHIP) short questionnaire for measuring oral healthrelated quality of life, and the current OHS was assessed objectively using Decayed, Missing, Filled Teeth/Surfaces (DMFT) index. Statistical analyses were done using structural equation modeling software.

Results: Our research showed relevant associations between HUDBI, DMFT, and OHIP. The relationship between HUDBI and OHIP was mediated by DMFT. Furthermore, HUDBI worked as a moderator between DMFT and OHIP. Thus, our study revealed a case for moderated mediation, which is usually ignored in similar research.

Conclusion: The "straightforward" causality between oral health-related behavior and the actual OHS must be considered with caution, as well as their impact on the oral health-related quality of life. Further research is needed to investigate the interaction between variables, the strength of the interrelations and the magnitude of their interactions, and the confidence that can be placed in these measurements, with respect to the general population and/or those lacking domain-specific education.

Keywords: moderated mediation, oral health, HUDBI, oral health-related quality of life, DMFT

\section{Introduction}

Oral health is a significant concern for the individual affected by various oral medical conditions because of its influence on the person's quality of life. Moreover, governments and other stakeholders are also affected in terms of socioeconomic costs. The severity and stringency of dealing with oral health-related problems is immediately evident when considering that oral diseases share the same risk factors as the four globally leading chronic diseases ${ }^{3}$ and that "renal, oral and eye diseases pose a major 
health burden for many countries". ${ }^{4}$ Moreover, even in modern times, the world faces the so-called 10/90 gap: as recently as 2004 , only $10 \%$ of the funding available to global health research was allocated to health problems that affect $90 \%$ of the world's population. ${ }^{5}$

This increasing interest for the implications of oral health led to the emergence of a new field of oral healthrelated quality of life. ${ }^{6}$ Recent research found significant and relevant associations between the quality of life and general oral health, ${ }^{7,8}$ and with specific oral health conditions, such as patients with malocclusions and orthodontic therapy, ${ }^{9-11}$ dental implants, ${ }^{12}$ removable dental prosthesis, ${ }^{13}$ or edentulism. ${ }^{14}$

However, the study of the relation between quality of life and oral health is by no means straightforward. First, [the] concepts of health and quality of life are elusive and abstract; while we know intuitively what they mean, they are difficult to define. ${ }^{15}$ Another reason is the multitude of factors that influence oral health. Environment, economics, and behavior are broadly regarded as the three main factors responsible for public health. ${ }^{16}$ This perspective has refined over the years to include biological, psychosocial, and the individual's behavior as increasingly more relevant and interrelated factors. ${ }^{17-19}$

Among the many studies that have investigated the relation between oral health and individuals' behavior, increasingly many use Kawamura's Dental Behavior Inventory, ${ }^{1}$ a self-reported list of preventive actions and behaviors for oral health. However, in contrast, much fewer studies bring together oral health, the related quality of life, and the individuals' behavior.

In this context, we were interested in investigating the relationships between oral health-related attitudes and behavior, oral health status (OHS), and oral health-related quality of life; their interactions; and the effects of their interactions. For instance, are the actions that a person takes in order to prevent oral health condition good predictors for the person's actual oral health? In addition, do individuals with better oral health also have higher levels of oral healthrelated quality of life?

We would expect that if a person takes good care of one's oral health, then that person has fewer oral health problems than those individuals who do not take any effective preventive actions. Intuitively, the causality between effective preventive action and actual oral health is somewhat implicitly presumed or speculated. We would also expect both the OHS and the attitudes and behavior to have some impact on the oral health-related quality of life of the person.
Therefore, our research aimed to develop and validate a scientific model, based on empirical evidence, which would accommodate the abovementioned directions of influence. Moreover, such a research model would require not only analyses of prediction (regressions) but also analyses of the potential effects of the interactions between the aforementioned variables and/or their ability to mediate and/or moderate one's effects upon another.

When not acknowledging or not including potential mediators in the model, the research design loses explanatory power with respect to its inner workings. Not including the potential moderator in the model leads to a predictable loss in the model's power to represent reality, since a certain measured effect is voided of its context (ie, missing those factors or conditions that make its measured direction and strength possible).

Thus, the scope of our research question had to include both analyses of mediation and/or moderation. If oral healthrelated attitude and behavior predicts and, it is implicitly assumed that, influences positively good OHS, then only OHS can be construed as a mediator in our three-variable model of interest. However, the possibility that either the oral health attitudes and behaviors or the OHS be a moderator in the other's relation with oral health-related quality of life is a subject open for investigation.

Therefore, two research objectives resulted. First, we investigated the OHS as a mediator between oral healthrelated attitudes and behaviors (as independent variable [IV]) and oral health-related quality of life (as dependent variable [DV]). Second, we investigated the role of OHS as a moderator and the role of oral health-related attitudes and behaviors as a moderator in our research model. Since, from a mathematical perspective, it is equal which one is construed as a moderator, what remains of practical interest is the magnitude and the significance of their interaction effect on the outcome variable. If either of these two analyses were to yield significant results, the possibility of a special case of moderator-mediator would have to be left open and investigated.

\section{Materials and methods Study design}

Our research employed a cross-sectional correlational design with selected predictor (IV) variables. In this context, we hypothesized a three-way relationship between oral healthrelated attitudes and behaviors, OHS, and oral health-related quality of life, in which oral health attitudes and behaviors served as independent or predictor variables, the OHS served 
as a mediator variable, and oral health-related quality of life served as an outcome/dependent or predictor variable.

In addition, in accordance with the mediator-moderator model developed by Preacher et al, ${ }^{20}$ we included oral healthrelated attitudes and behaviors as a moderator for the relationship between OHS (as IV) and oral health-related quality of life (as DV). We made this decision because the direction of causality is intrinsically implied by the supposition that preventive oral health actions (are performed in order to) lead to good oral health.

\section{Procedure}

Our study was approved by the decision 496/09.02.2016 of the Ethical Board of Iuliu Haţieganu University of Medicine and Pharmacy, Cluj-Napoca, Romania and observed all ethical guidelines of the decision. Participation in the study was voluntary and accepted based on informed written consent. Data collection was made via paper and pencil questionnaires. The questionnaires were distributed, completed, and collected during a school session, and the clinical evaluation for the current OHS was done in the school's clinical setting.

A single, qualified investigator completed the clinical examination. The investigator placed the subjects in a supine position, using artificial white light, with a dental explorer, a flat-surface mouth mirror, and compressed air in the Department of Odontology, Endodontics, Cardiology, Oral Pathology, Faculty of Dental Medicine, "Iuliu Haţieganu" University of Medicine and Pharmacy, Cluj-Napoca, Romania, under aseptic conditions.

With respect to training and calibration, before conducting the study, the investigator was calibrated at the school's specialty department in order to limit examiner variability, and kappa value was found to be 0.87 , which was satisfactory. In order to assess the intra-examiner reliability, the investigator applied the Decayed, Missing, Filled Teeth/ Surfaces (DMFT) index to 30 selected subjects and recorded the findings. The same subjects were randomly examined again in the following days by the investigators. The intrarater agreement was $80 \%$, which reflected a high degree of conformity in observation. ${ }^{3,21}$

\section{Participants}

The participants included $\mathrm{N}=191$ students enrolled in the fourth and sixth years of study at the Dentistry School of the Medicine and Pharmacology, University of Cluj-Napoca. In all, 50 (26.18\% of the total number of 191 participants) and $69(36.13 \%)$ participants were female in the fourth and sixth years of study, respectively, whereas an equal number of $36(18.85 \%$ of the total number) participants were male in both fourth and sixth years of study. The participants, mean age was $M_{\mathrm{AGE}}=24.12$ years and standard deviation (SD) was $2.30\left(M_{\text {Female }}=23.78, \mathrm{SD}=1.83\right.$, and $M_{\text {Male }}=24.67$, $\mathrm{SD}=2.85$ ) for the entire group.

Because the participants were recruited from the fourth and sixth years of study, they constitute a convenience sample. The inclusion criteria were as follows: dental student, $>18$ years of age, good general health without serious medical conditions necessitating hospitalization in the past 3 months and absence of conditions requiring psychological counseling or the consumption of antidepressants or anxiolytics that can directly affect psychological health and the quality of life. All participants completed successfully the self-reported measures and the clinical evaluation, ie, there were no discarded responses and no excluded participants.

\section{Measures}

\section{Oral health-related attitudes and behavior}

The respondents' specific oral health-related behavior and attitudes were measured using Hiroshima University Dental Behavior Inventory (HUDBI), developed by Kawamura. HUDBI measures the respondents' agreement to 20 dichotomous ("yes" and "no") items formulated as statements (for instance, "I brush each of my teeth carefully" or "I think I can clean my teeth well without using toothpaste"). Items are rated with " 0 " or " 1 ", as the respondent expresses disagreement ("0") or agreement ("1"), and then are summed to yield a total score. Only 12 out of the total of 20 items are scored, which are given as follows: items 2, 6, 8, 10, 14, and 15 are rated " 1 " when disagreement is expressed, and items $4,9,11,12,16$, and 19 are rated " 1 " when agreement is expressed. The higher the scores on HUDBI, the higher the respondent's adequacy to good self-care oral health-related attitudes and behaviors.

The scale's reliability was reported as good in the existing research. ${ }^{20-29}$ In our study, we used the Romanian version of HUDBI, which was also used in previous studies. ${ }^{30,31}$ In our study, HUDBI's internal consistency, resulting after performing categorical principal component analysis (CATPCA) in SPSS, was $\alpha_{\text {Cronbach }}=0.81$.

\section{Oral health-related quality of life}

Oral health-related quality of life was measured using a short 14 items adapted version of the Oral Health Impact Profile (OHIP), developed by Slade ${ }^{2}$ in 1997 and derived 
from the original 49 items instrument designed in $1994 .{ }^{32}$ The Romanian version, OHIP-14 Ro is a 14-item, 5-step Likert scale measuring the self-reported frequency of discomfort symptoms such as functional limitation, physical pain, psychological discomfort, psychological disability, social disability, and perceived handicap. OHIP-14 Ro was previously used in studies involving Romanian individuals, and it showed good internal consistency $\left(\alpha_{\text {Cronbach }}=0.88\right)$ and strong and statistically significant associations with objectively measured oral health. ${ }^{33}$ In our study also, the internal consistency was $\alpha_{\text {Cronbach }}=0.88$.

\section{OHS}

The current OHS was assessed objectively via the use of DMFT index, designed by Klein et $\mathrm{al}^{34}$ and indicated by the World Health Organization (WHO) as a diagnostic criterion for decayed, missing, and filled teeth and surfaces. ${ }^{3}$ The WHO has standardized its use in oral health surveys for describing dental status in children, adults, and the elderly. The DMFT index is used for estimating the prevalence of dental caries in the permanent dentition and is represented by the total number of teeth that are decayed (D), missing (M) or filled (F) for an individual.,35 The DMFT score per individual can range from 0 to 32 since 1987 when the WHO started recommending that the third molars should be included in the scoring. ${ }^{3}$

\section{Statistical analyses}

The required statistical analyses were done using structural equation modeling (SEM) software such as IBM's SPSS Amos ver. 22 and IBM's SPSS Amos ver. 21 and replicated with the PROCESS macro module developed by Hayes, ${ }^{36}$ which includes the Johnson-Neyman technique ${ }^{37}$ for probing regions of significance. One reason why we performed SEM is that it allows testing all influence pathways simultaneously as opposed to multiple regression, which has a sequential approach.

Another reason for using both IBM's Amos and SmartPLS was the novelty of the research. IBM Amos is a covariancebased modeling software, whereas SmartPLS relies on partial least squares. Both programs employ methods that have complementary strength, which cover sensitive areas of our research. According to Hair et al, ${ }^{38}$ partial least squares structural equation modeling (PLS-SEM) is best suited for predicting key target constructs and exploration or extension of existing theory, whereas for theory testing, theory confirmation, or comparison of alternative theories, covariance-based SEM is best suited. ${ }^{38}$
Moreover, because the adequate sample size is a controversial issue in SEM, we doubled the use of IBM SPSS Amos with that of SmartPLS, because the latter can well handle small samples and non-normal distributed data. ${ }^{39,40}$ However, because we needed to probe a special case of moderator-mediator, Amos was required as well in order to estimate the significance of the pathways and not only the effects of the interaction between terms. No cases of aberrant or missing data were encountered.

\section{Results}

\section{Relevant associations between the main variables}

A preliminary correlation analysis for HUDBI, DMFT, and OHIP showed statistically significant associations between each of these three variables. Pearson's correlation coefficients were $\rho_{\text {HUDBI-DMFT }}=-0.15$ (at $p=0.035$ ), $\rho_{\text {HUDBI-OHIP }}=-0.22$ (at $p=0.003$ ), and $\rho_{\text {DMFT-OHIP }}=0.26$ (at $p<0.001$ ). Regression analyses confirmed that there were statistically significant relations between HUDBI and DMFT, between HUDBI and OHIP, and between DMFT and OHIP. Individually (unmediated and un-moderated), both HUDBI and DMFT predicted OHIP: $\beta_{\text {HUDBI-OHIP }}=-22$, $p=0.002$, and $\beta_{\text {DMFT-OHIP }}=0.26, p<0.001$.

\section{Mediation analysis}

The mediation model consisted of HUDBI as an IV, DMFT as a mediator, and OHIP as a DV. The mediation model was developed in IBM SPSS Amos ver. 21 and in SmartPLS $3 .{ }^{41}$ After mediation, HUDBI continued to significantly predict DMFT, although at a smaller significance threshold $(p=0.034)$ than it did for OHIP $(p=0.01)$, and at a smaller strength of association, $\beta_{\text {HUDBI-DMFT }}=-0.15$, whereas $\beta_{\text {HUDBI-OHIP }}=-0.18$ (Figure 1). For comparison, previous

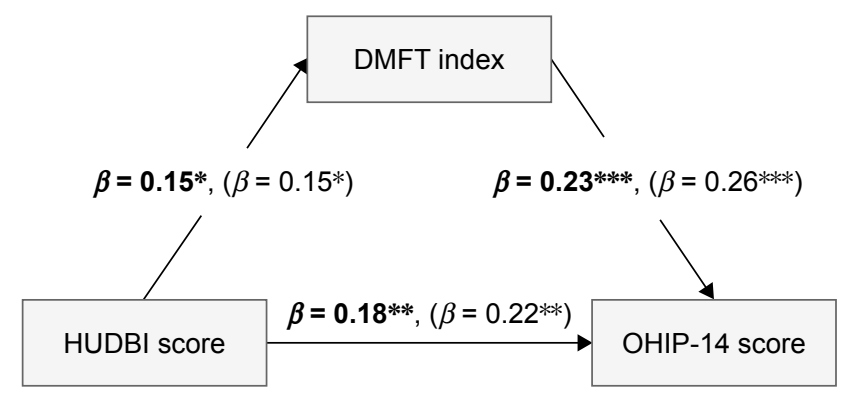

Figure I Strength of the associations between HUDBI, DMFT, and OHIP-I4 (after the mediation by DMFT in bold text and before mediation by DMFT in brackets).

Note: The significance levels $0.05,0.01$, and 0.001 are denoted by a *, **, and ***, respectively.

Abbreviations: HUDBI, Hiroshima University Dental Behavior Inventory; DMFT, Decayed, Missing, Filled Teeth/Surfaces; OHIP, Oral Health Impact Profile. 


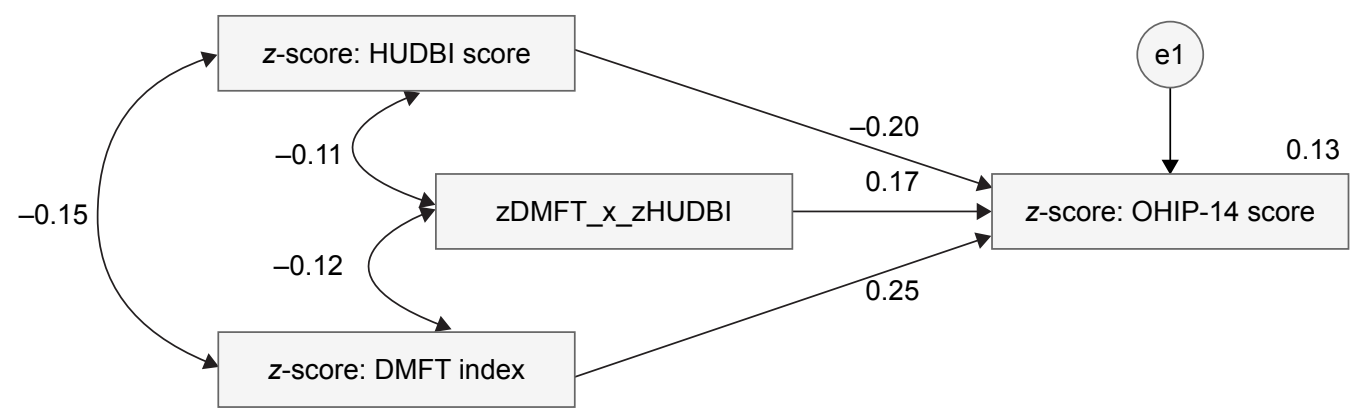

Figure 2 Moderation analysis for HUDBI and DMFT predicting OHIP-I4 and their interaction effect using z-standardized data.

Abbreviations: HUDBI, Hiroshima University Dental Behavior Inventory; DMFT, Decayed, Missing, Filled Teeth/Surfaces; OHIP, Oral Health Impact Profile.

regression weights, before mediation, were $\beta_{\text {DMFT-OHIP }}=0.26$ and $\beta_{\text {HUDBI-OHIP }}=-0.22$.

The mediation analysis was replicated in SPSS using PROCESS to perform mean centering, bootstrapping, and Sobel's test (see the output of the mediation analysis in the Supplementary material section for details). According to the mediation analysis performed with PROCESS, path $c$, between $X$ and $Y$, was still statistically significant $(p=0.004)$ and the $95 \%$ confidence interval (CI) ranged from -0.36 to -0.07 , which did not include zero.

One widely accepted condition when testing for mediation was that the direct path $(X$ to $Y)$ is "significantly decreased" after the mediation. ${ }^{42}$ While identifying a decrease is straightforward, what is "significant" is not. Sample size, for instance, is one of the factors that influence "significance" of association in correlational studies - ie, a smaller effect in a larger sample size can have stronger significance than a larger effect in a smaller sample size. This is immediately apparent due to the decrease in CI's range with the increase in the sample size.

In our study, the recorded decrease was indicative of existence of mediation. However, the rather small magnitude of this decrease, together with the fact that the direct effect (from HUDBI to OHIP) remained highly statistically significant after mediation $(p<0.05)$, indicated that HUDBI is not a strong mediator for the model. In addition, it is worth noting that one of the assumptions of mediation is that no $X^{*} M$ interaction takes place in the model. ${ }^{43}$ Thus, a moderation analysis was required in the next step to investigate if such an interaction existed.

\section{Moderation analysis}

The moderation model considered DMFT as IV and OHIP as DV with HUDBI as the moderator (Figures 2 and 3). According to the results after applying PROCESS procedure for SPSS of Hayes, ${ }^{36}$ which includes the Johnson-Neyman technique ${ }^{44}$ the overall moderation model had significant effects $F(3,187)=5.93, p<0.001$. However, the overall interaction effect was not significant: $\mathrm{CI}=-0.26$ to 0.06 (the $\mathrm{CI}$ includes the zero value). Therefore, we further used the Johnson-Neyman ${ }^{37}$ technique to probe for interaction and to identify ranges of values of the moderator for which the interaction effect is significant.

One such region of significant moderation was identified, for values of HUDBI (in $z$-scores) from -4.49 to 0.52 . The moderation effect is graphically depicted in Figures 4 and 5 and shows that the moderator HUDBI strengthened the positive relation between DMFT and OHIP for the regions of significance indicated by the Johnson-Neyman technique.

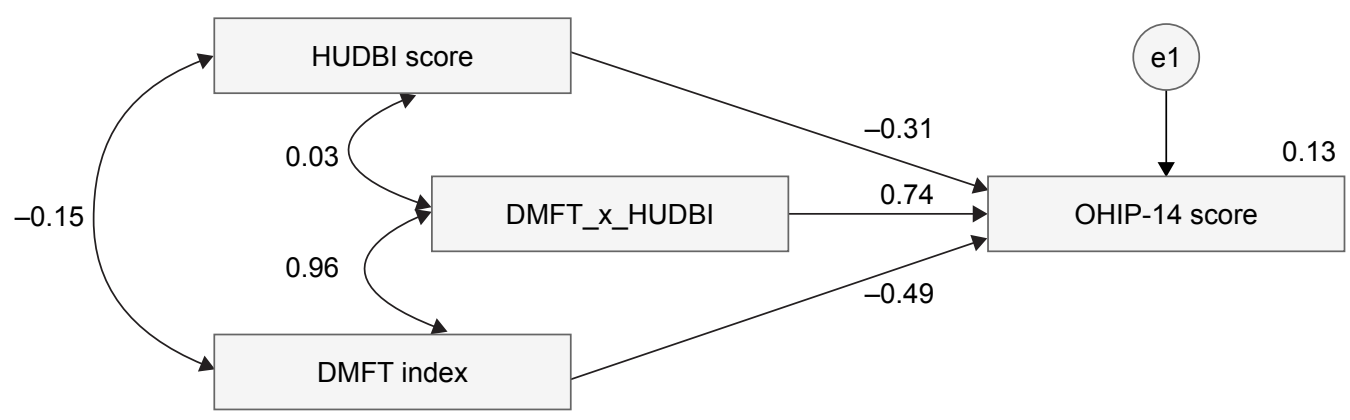

Figure 3 Moderation analysis for HUDBI and DMFT predicting OHIP-I4 and their interaction effect using originally scaled scores.

Abbreviations: HUDBI, Hiroshima University Dental Behavior Inventory; DMFT, Decayed, Missing, Filled Teeth/Surfaces; OHIP, Oral Health Impact Profile. 


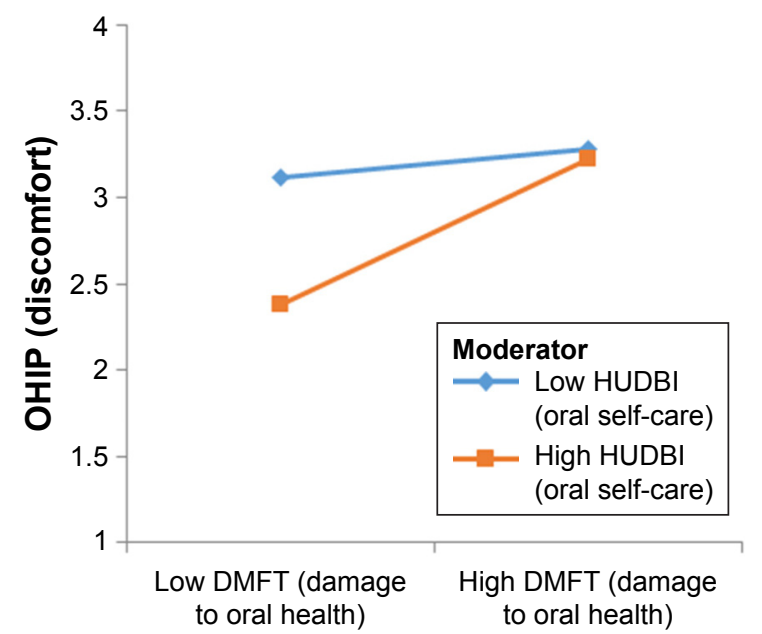

Figure 4 Plot for mean interaction indicative of $\mathrm{HUDBI}$ as a moderator, strengthening the positive relationship between DMFT and OHIP-I4.

Abbreviations: HUDBI, Hiroshima University Dental Behavior Inventory; DMFT, Decayed, Missing, Filled Teeth/Surfaces; OHIP, Oral Health Impact Profile.

In our moderation analysis, we used $z$-scores for all three main variables because, although for cases other than extreme multicollinearity, there should be no difference in the $p$-value for the interaction term, regardless of whether $z$-scores or the original scores are used. ${ }^{45,46} z$-scoring (or $z$-standardization) of data, as opposed to using original scaled data, has the advantage of allowing "apples-to-apples" comparison for the interaction terms and regression coefficients. ${ }^{47}$ Mean centering is also done implicitly in Hayes' PROCESS macro for moderated regressions in SPSS. ${ }^{36,48}$

As such, both alternatives - ie, for moderation with $z$-standardized data and, respectively, for moderation with raw data - were reported (Figures 2 and 3). It is important to observe that while the correlation between the original

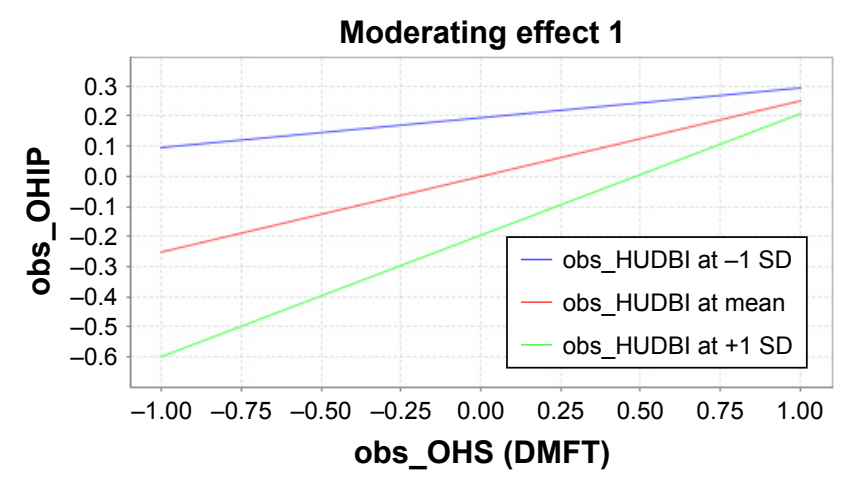

Figure 5 Plot for mean interaction indicative of HUDBI as a moderator, strengthening the positive relationship between DMFT and OHIP-I4 using the variables' standardized values or z-scores.

Note: The blue, red, and green lines indicate the evolution of OHIP with OHS scores for $-I$ SD scores of HUDBI, mean scores of HUDBI, and +I SD scores of HUDBI, respectively.

Abbreviations: HUDBI, Hiroshima University Dental Behavior Inventory; DMFT, Decayed, Missing, Filled Teeth/Surfaces; OHIP, Oral Health Impact Profile; SD, standard deviation. variables and their interaction product may be much larger than the correlation between their corresponding normalized data, the statistical interpretation of their interaction effect is the same in both models. ${ }^{49,50}$

The moderation analysis using the Johnson-Neyman techniques identified a range of values for the interaction between HUDBI and DMFT, at which significant moderation takes place. More specifically, for low levels of oral health, there is little difference in terms of perceived and self-reported oral health-related quality of life between people who have better levels of oral attitudes and behaviors and those with lower levels. However, for good levels of oral health, there is a bigger difference between those who have better oral health attitudes and behavior and those who do not, in terms of self-reported oral health-related quality of life (Figures 4 and 5).

\section{Moderated mediation model}

The analysis for the moderated mediation case was performed using IBM SPSS Amos ver. 22 and SmartPLS 3 (Figures 6-8), which yielded similar results. Because SmartPLS utilizes $z$-scores in computing the interaction effects, we used the same type of standardized scores for all three variables in IBM SPSS Amos. Statistically significant effects were recorded for all four pathways of interests: 1) $\left.\beta_{\text {zHUDBI - zOHIP }}=-0.20, p<0.05 ; 2\right) \beta_{\text {zDMFT }- \text { zOHIP }}=0.25$, $p<0.001$; 3) $\beta_{\text {(zHUDBI } *_{\text {zDMFT }) \text { zOHIP }}}=0.17, p<0.05$; and 4) $\beta_{\mathrm{zHUDBI}-\mathrm{zDMFT}}=-0.15, p<0.05$.

Because HUDBI's influence on OHIP is only weakly diminished and is still statistically significant even after being mediated by DMFT, it suggests that more favorable oral health attitudes and behaviors lead to a better oral health-related quality of life, even if the actual OHS is affected. Moreover, based on the moderation analysis results, considered also in the moderated mediation model, it appears that the impact of oral health attitudes and behaviors is substantially better for those with relatively good OHS, and it makes less of a difference for people with a more compromised OHS. Most importantly, the results from the moderated mediation model suggest that both effects happen simultaneously.

It is worth noting that the three main variables modeled in our study do not have a great degree of relatedness. In regular psychological or social research, most of the modeled constructs are usually related. For instance, measuring the mediation effect of state anxiety in the relation between stress and depression makes very good intuitive sense, since all three variables stand for psychological constructs, and these constructs are more or less related. This is an important 


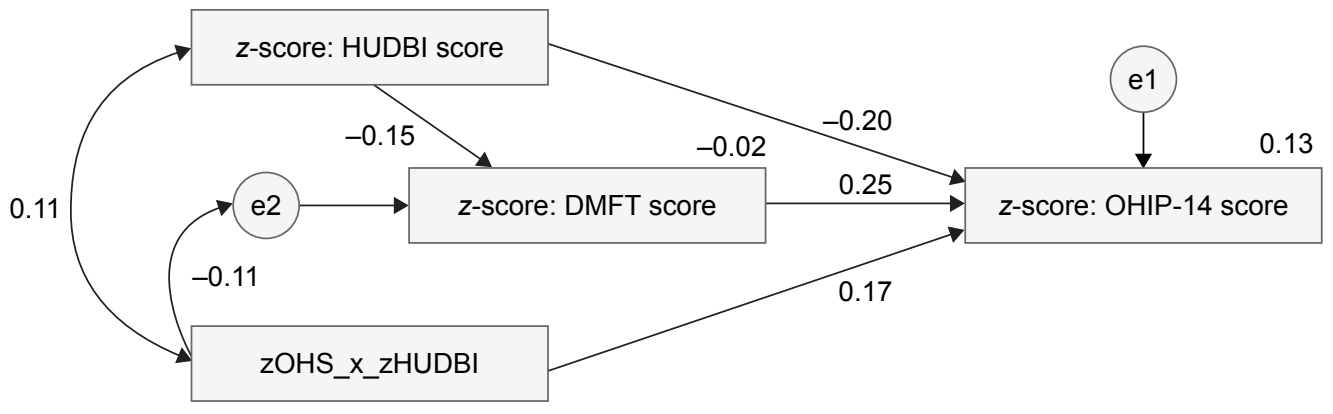

Figure 6 Model of moderated mediation.

Abbreviations: HUDBI, Hiroshima University Dental Behavior Inventory; DMFT, Decayed, Missing, Filled Teeth/Surfaces; OHIP, Oral Health Impact Profile.

caveat to consider when interpreting results based on associations between variables, since association is extremely sensitive to variations in the sample properties, such as size and noise..$^{51-55}$

\section{Discussion}

We developed and tested a complex mediation-moderation model in which the actual OHS mediated and moderated the relation between the individual's oral health-related preventive behavior and the perceived oral health-related quality of life. In the absence of explicit empirical support from previous research, we had to consider both mediation and moderation analyses in our study. We performed a series of mediation and moderation analyses using both "classical" hierarchical regression and the SEM approach, which is suggested by some authors as a better alternative. ${ }^{56,57}$ Our analyses and the findings provided evidence for the need to consider this type of model.

More specifically, for instance, if one uses OHIP to measure detrimental effects to a person's quality of life resulting

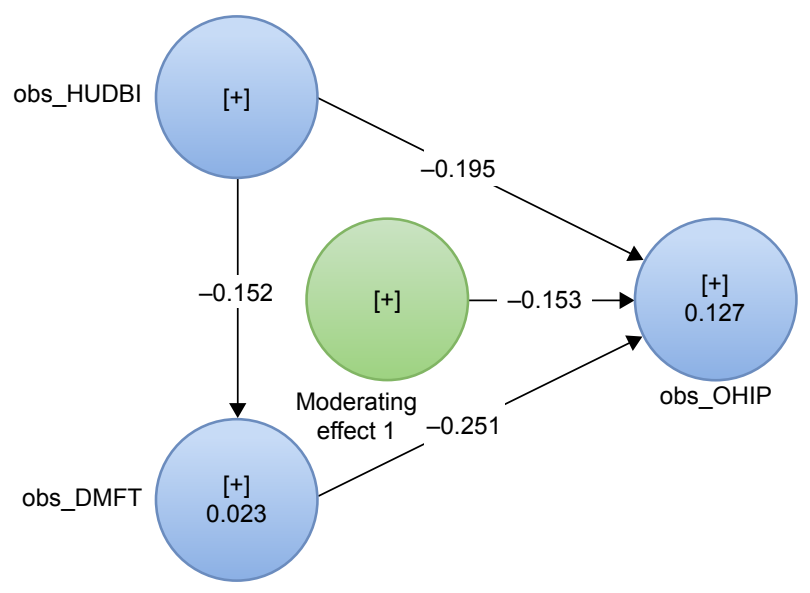

Figure 7 Model of moderated mediation with partial least squares.

Abbreviations: HUDBI, Hiroshima University Dental Behavior Inventory; OHIP, Oral Health Impact Profile; DMFT, Decayed, Missing, Filled Teeth/Surfaces. from a poor OHS, it is implicitly assumed that a poor OHS leads to specific and consistent corresponding scores on OHIP. As logical as this may seem, and making a note that ours was not a study regarding the validity (convergent or discriminant) of OHIP or of any other of the instruments used here, there are still conceptual issues to address. The most pressing of those is the relatedness of the measured constructs. Modeling relatively unrelated constructs requires at least considering the possibility of moderation, rather than that of mediation.

Moderation does not have to meet the same stringency as mediation regarding the conceptual relatedness. Humidity may moderate the relation between temperature and headaches, without having to be conceptually related to either of the other two. It is important to note in our model that the three main variables were rather conceptually weakly related. Oral health-related quality of life measured a psychological construct, whereas the OHS measured a physical, observable state of facts. Finally, the oral health attitude and behavior questionnaire accounts for a set of self-reported

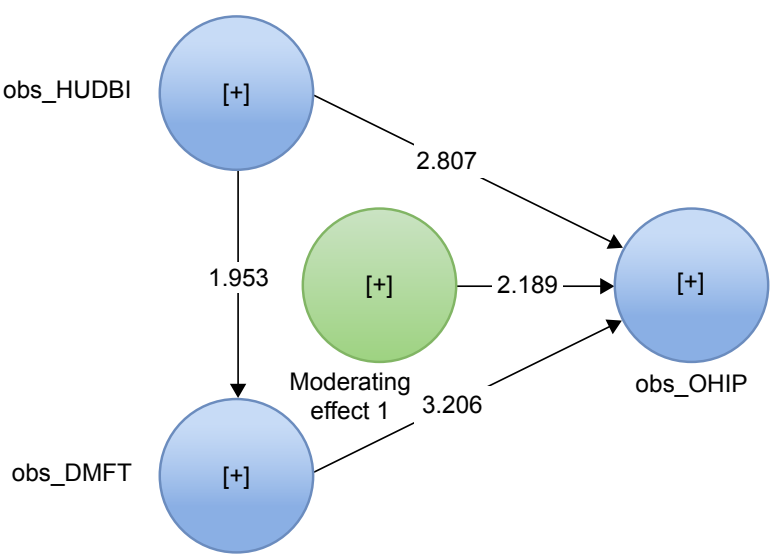

Figure 8 Model of moderated mediation using partial least squares, after bootstrapping.

Abbreviations: HUDBI, Hiroshima University Dental Behavior Inventory; OHIP, Oral Health Impact Profile; DMFT, Decayed, Missing, Filled Teeth/Surfaces. 
learned activities, and in a much lesser degree for the individual's psychological assessments that stand behind these activities.

Although the minimal methodological conditions for analyzing OHS as a mediator in the model were met, based on our study results, OHS was not an "authentic" or "true" mediator. More specifically, the relation between HUDBI and OHIP was still significant and the decrease in strength was small, after mediation from DMFT. This according to Baron and Kenny ${ }^{42}$ points to the need to consider other mediators in the model. Furthermore, more comprehensive research may consider the role of placebo or self-suggestion mechanisms, when trying to explain why there is still an impact from oral self-care to the state of well-being, even after controlling for the actual OHS. It is also not only possible but also reasonable to assume that the self-care actions contribute positively to increase comfort and well-being, even when the OHS is affected.

Our measure of choice for the OHS, albeit valid and objective, may not be indicative of everything that is understood by oral health means/stands. For instance, bad breath or halitosis can be caused by a variety of conditions, and not only by oral health conditions. Decayed teeth and related infections are not the only possible causes of tooth pain. It is also possible that a person evaluated with a high DMFT and, thus, considered as having a poor OHS does not feel discomfort if his/her condition is not acute or is not painful/ disturbing enough. As such, such an individual would not report higher scores on OHIP. Moreover, there is a higher percentage of patients with oral health conditions among poor and disadvantaged groups within the population all over the globe. Some of the risk factors that were identified for oral health conditions are unhealthy diet, tobacco use, harmful alcohol use, poor oral hygiene and social determinants. ${ }^{58-60}$

Therefore, while the results were both significant and suggestive and confirmed our initially hypothesized model, they also suggest that other factors are also involved in determining one's state of oral health-related quality of life. As such, searching only for mediation, although intuitively appealing, is rather weakly founded. Moderation, on the other hand, makes much more sense, both from theoretical grounds and statistically. Even more so, our results led us toward a special case of moderated mediation. As Baron and Kenny ${ }^{42}$ suggested,

if the residual path $c$ (ie, between $X$ and $Y$ ) is not zero, this indicates the operation of multiple mediating factors.

[...] a more realistic goal may be to seek mediators that significantly decrease path $c$ rather than eliminating the relation between the independent and dependent variables altogether. $^{42}$

\section{Limitations and future research}

Our study involved (only) $\mathrm{N}=191$ people, all fourth- and sixth-year dentistry students, which amounts to a very narrow demographic segment. Moreover, the participants shared a lot of domain-specific knowledge and many sociodemographic characteristics. Dental students should not be taken as representatives for a heterogeneous population because their interest in the dentistry domain might predispose them to a better understanding, better approach, and behaviors that will eventually lead to better results both as far as HUDBI and DMFT are concerned. Improvement in dental health attitudes and behaviors in the dental students may be attributed to their incentive toward a dental career. ${ }^{61}$

Therefore, studies with much more diverse participants would be useful in order 1) to investigate the proposed research model and its parameters into a more diverse population and 2) to gain power to extend the findings to the general population.

To a certain extent, both mediation and moderation happened. Furthermore, our data supported and were indicative of a special case of moderated mediation. However, is the relation between our three research variables one of mediator-moderator? It is still too early to make such an assertion. Our results indicate this possibility and provide explicit empirical evidence for our particular research.

In addition, the interpretation of the magnitude of the moderation interaction and that of the mediation's effect are still unclear. The current state of research does not offer a relevant comparative framework, which allows for the interpretation of effects' magnitude.

Further research is needed to investigate and provide more confidence as to 1) if the identified interaction between oral health-related quality of life, OHS, and oral health attitudes and behaviors represents indeed a special case of moderated mediation; 2) what is the strength of the interrelations between the aforementioned variables and the magnitude of their interactions; and 3) what is the confidence that can be placed in these measurements.

Because human behavior is especially complex in terms of causes and interdependencies, it is mandatory to interpret the results within the context of the possibility of many other mediators and confounded variables not included in the model. ${ }^{62}$ Our results support this need not only from a technical/statistical standpoint - ie, gain explanatory power by increasing the model's complexity - but also because 
each of our variables can reasonably be affected by a cohort of moderators.

Future related studies can make good use by incorporating several variables. For instance, age and (specific) education can be investigated as to their effect on HUDBI, whereas DMFT can also be placed in a broader context of oral health. Not in the least, OHIP is susceptible to be influenced by other personality traits, such as trait anxiety, optimism, determination, stress resilience, and coping potential. With respect to more particular subjective behavior, future studies could consider the influence of people's preference to use electric versus manual toothbrushing would be useful and of interest.

\section{Conclusion}

While clinicians aim to improve patients' oral healthrelated quality of life through performing medical treatments, clinicians and policymakers emphasize the role played by other factors - such as attitudes and behaviors - in the broad construct of self-perceived oral health-related quality of life. Scientific studies have showed that individuals with a higher dental specific education achieve better scores for HUDBI, and therefore, the need for specific education with respect to oral health-related practices should be emphasized, both in the dental practice and as a general public oral health policy. ${ }^{63-65}$

Eitner et $\mathrm{al}^{66}$ concluded that in the case of dental implant therapy while the patients recorded a decrease in oral healthrelated quality of life, the physicians did not account for this decrease during the treatment. Therefore, physicians should forewarn their patients regarding the likely decrease in oral health-related quality of life in order not to compromise the likelihood of complete implant therapy success.

All measures used in the study account for the spot state. The values for all three variables may change with time. A special case may occur for individuals undergoing long and multi-visit treatment - such as orthodontic therapy or dental implants followed by prosthodontic treatment. While the dental procedures aim to improve the OHS, the patient may experience a drop in the self-perceived oral health-related quality of life during the treatment period. ${ }^{67,68}$ We believe that our study brings concrete evidence that clinicians and dental educators could benefit from understanding and incorporating in their professional practice the inter-relations between the actual oral health, the individuals' self-care practices, and their perceived impact.

\section{Acknowledgment}

The authors would like to extend their gratitude to all participants who volunteered to be part of this study.

\section{Author contributions}

Alexandra Vigu designed the study, performed the experiments and data collection, and carried out manuscript writing. Dorin Stanciu performed methodology (research design, hypotheses testing, statistical data processing), manuscript writing, and proofreading. Lucia Maria Lotrean was involved in data interpretation and writing the article. Radu Septimiu Campian performed main research idea, hypothesis, and proofreading. All the authors were involved in revising the article critically for important intellectual content and have approved the version to be published.

\section{Disclosure}

The authors report no conflicts of interest in this work.

\section{References}

1. Kawamura M. The relationship between perceptions of oral health and oral status in adults. J Hiroshima Univ Dent Soc. 1988;20: 273-286.

2. Slade GD. Derivation and validation of a short-form oral health impact profile. Community Dent Oral Epidemiol. 1997;25(4):284-290.

3. World Health Organization. Oral Health Surveys: Basic Methods. Geneva: World Health Organization; 2013.

4. FDI World Dental Federation. Oral Health and the United Nations Political Declaration on NCDs. A Guide to Advocacy. 2011. Available from: https:// www.fdiworlddental.org/sites/default/files/media/images/oral_health_and_ un_political_dec_on_ncds.pdf. Accessed March 17, 2018.

5. Petersen PE. Priorities for research for oral health in the 21st centurythe approach of the WHO Global Oral Health Programme. Community Dent Health. 2005;22(2):71-74.

6. Cohen LK, Slade G. The emerging field of oral health-related quality of life outcomes research. Measur Oral Health Qual Life. 1996:1-10.

7. Brennan DS, Singh KA, Spencer AJ, Roberts-Thomson KF. Positive and negative affect and oral health-related quality of life. Health Qual Life Outcomes. 2006;4(1):1-10.

8. Brennan DS, Teusner DN. Oral health impacts on self-rated general and oral health in a cross-sectional study of working age adults. Community Dent Oral Epidemiol. 2015;43(3):282-288.

9. Cutroneo G, Piancino MG, Ramieri G, et al. Expression of musclespecific integrins in masseter muscle fibers during malocclusion disease. Int J Mol Med. 2012;30(2):235-242.

10. Cavuoti S, Matarese G, Isola G, Abdolreza J, Femiano F, Perillo L. Combined orthodontic-surgical management of a transmigrated mandibular canine. Angle Orthod. 2015;86(4):681-691.

11. Zhou Y, Wang Y, Wang X, Volière G, Hu R. The impact of orthodontic treatment on the quality of life a systematic review. BMC Oral Health. 2014;14(1):66.

12. Alzarea BK. Assessment and evaluation of quality of life (OHRQoL) of patients with dental implants using the oral health impact profile (OHIP-14) - a clinical study. J Clin Diagn Res. 2016;10(4):57-60.

13. Al-Imam H, ÖZhayat EB, Benetti AR, Pedersen AML, Gotfredsen K. Oral health-related quality of life and complications after treatment with partial removable dental prosthesis. J Oral Rehabil. 2016;43(1): 23-30.

14. Batista MJ, Procopio Lawrence H, da Luz Rosário de Sousa M. Impact of tooth loss related to number and position on oral health quality of life among adults. Health Qual Life Outcomes. 2014;12(1):165-174.

15. Slade GD. Measuring oral health and quality of life. Chapel Hill, NC: University of North Carolina; 1997.

16. McKeown T. The Role of Medicine: Dream, Mirage or Nemesis? Princeton, NJ: Princeton University Press; 1979. 
17. Anderson R. Health Behaviour Research and Health Promotion. Oxford, NY: Oxford University Press; 1988.

18. Andersen R, Marcus M, Mahshigan M. A comparative systems perspective on oral health promotion and disease prevention. In: Cohen LK, Gift HC, editors. Disease Prevention and Oral Health Promotion: SocioDental Sciences in Action. Copenhagen: Munksgaard; 1995:307-340.

19. Andersen RM. Revisiting the behavioral model and access to medical care: does it matter? J Health Soc Behav. 1995;36(1):1-10.

20. Preacher KJ, Rucker DD, Hayes AF. Addressing moderated mediation hypotheses: theory, methods, and prescriptions. Multivariate Behav Res. 2007;42(1):185-227.

21. McHugh ML. Interrater reliability: the kappa statistic. Biochem Med (Zagreb). 2012;22(3):276-282.

22. Doğan B. Differences in oral health behavior and attitudes between dental and nursing students. J Marmara Univ Inst Health Sci. 2013;3(1): $34-40$.

23. Jaramillo JA, Jaramillo F, Kador I, et al. A comparative study of oral health attitudes and behavior using the Hiroshima University-Dental Behavioral Inventory (HU-DBI) between dental and civil engineering students in Colombia. J Oral Sci. 2013;55(1):23-28.

24. Kawamura M, Yip HK, Hu Y, Komabayashi T. A cross-cultural comparison of dental health attitudes and behaviour among freshman dental students in Japan, Hong Kong and West China. Int Dent J. 2001;51(3): 159-163.

25. Kim K-J, Komabayashit T, Moon S-E, Goo K-M, Okada M, Kawamura M. Oral health attitudes/behavior and gingival self-care level of Korean dental hygiene students. J Oral Sci. 2001;43(1):49-53.

26. Komabayashi T, Kwan SYL, Hu D-Y, Kajiwara K, Sasahara H, Kawamura M. A comparative study of oral health attitudes and behaviour using the Hiroshima University - Dental Behavioural Inventory (HU-DBI) between dental students in Britain and China. J Oral Sci. 2005;47(1):1-7.

27. Rong WS, Wang WJ, Yip HK. Attitudes of dental and medical students in their first and final years of undergraduate study to oral health behaviour. Eur J Dent Educ. 2006;10(3):178-184.

28. Sato M, Camino J, Oyakawa HR, et al. Effect of dental education on Peruvian dental students' oral health-related attitudes and behavior. $J$ Dent Educ. 2013;77(9):1179-1184.

29. Yildiz S, Dogan B. Self reported dental health attitudes and behaviour of dental students in Turkey. Eur J Dent. 2011;5(3):253-259.

30. Dumitrescu A, Kawamura M, Sasahara H. An assessment of oral self-care among Romanian dental students using the Hiroshima University - Dental Behavioural Inventory. Oral Health Prev Dent. 2007; 5(2):95-100.

31. Truță RI, Milicescu V. Evaluarea atitudinii şi comportamentului faţă de propria sănătate orală la un grup de studenţi la medicină dentară [The assessment of oral health attitudes and behaviors in a group of dental students]. Rev Română Stomatol. 2015;LXI(1):100-104. Romanian.

32. Slade GD, Spencer AJ. Development and evaluation of the oral health impact profile. Community Dent Health. 1994;11(1):3-11.

33. Slusanschi O, Moraru R, Garneata U, Mircescu G, Cuculescu M, Preoteasa E. Validation of a Romanian version of the short form of the oral health impact profile (OHIP-14) for use in an urban adult population. Oral Health Prev Dent. 2013;11(3):235-242.

34. Klein H, Palmer CE, Knutson JW. Studies on dental caries: I. Dental status and dental needs of elementary school children. Public Health Reports (1896-1970). 1938;25(10):103-1705.

35. Larmas M. Has dental caries prevalence some connection with caries index values in adults? Caries Res. 2010;44(1):81-84.

36. Hayes AF. PROCESS: A versatile computational tool for observed variable mediation, moderation, and conditional process modeling. 2012. Available from: http://www.afhayes.com/public/process2012.pdf.

37. Johnson PO, Neyman J. Tests of certain linear hypotheses and their application to some educational problems. Statist Res Mem. 1936:57-93.

38. Hair JF, Ringle CM, Sarstedt M. PLS-SEM: indeed a silver bullet. J Market Theory Pract. 2011;19(2):139-152.
39. Aguirre-Urreta M, Rönkkö M. Sample size determination and statistical power analysis in PLS using R: an annotated tutorial. Commun Assoc Inf Syst. 2015;36(1):3.

40. Hair JF, Hult GTM, Ringle C, Sarstedt MA. Primer on Partial Least Squares Structural Equation Modeling (PLS-SEM). Thousand Oaks, CA: SAGE; 2016.

41. SmartPLS 3 [computer program]. Boenningstedt: SmartPLS GmbH; 2015.

42. Baron RM, Kenny DA. The moderator-mediator variable distinction in social psychological research: conceptual, strategic, and statistical considerations. J Pers Soc Psychol. 1986;51(6):1173-1182.

43. Fairchild AJ, MacKinnon DP. A general model for testing mediation and moderation effects. Prev Sci. 2009;10(2):87-99.

44. Hayes AF, Matthes J. Computational procedures for probing interactions in OLS and logistic regression: SPSS and SAS implementations. Behav Res Methods. 2009;41(3):924-936.

45. Echambadi R, Hess JD. Mean-centering does not alleviate collinearity problems in moderated multiple regression models. Market Sci. 2007; 26(3):438-445.

46. Kromrey JD, Foster-Johnson L. Mean centering in moderated multiple regression: much ado about nothing. Educ Psychol Meas. 1998;58(1): $42-67$.

47. Dawson JF. Moderation in management research: what, why, when, and how. J Bus Psychol. 2014;29(1):1-19.

48. Hayes AF. Introduction to Mediation, Moderation, and Conditional Process Analysis: A Regression-Based Approach. New York, NY: Guilford Press; 2013.

49. Gatignon H, Vosgerau J. Stochastic moderated regression: an efficient methodology for estimating parameters in moderated regression. 2006. Available from: https://flora.insead.edu/fichiersti_wp/ inseadwp2006/2006-17.pdf

50. Gatignon H, Vosgerau J. Moderating Effects: The Myth of Mean Centering. Fontainbleau Cedex: INSEAD. 2005.

51. Schönbrodt FD, Perugini M. At what sample size do correlations stabilize? J Res Pers. 2013;47(5):609-612.

52. Kelley K, Maxwell SE. Sample size for multiple regression: obtaining regression coefficients that are accurate, not simply significant. Psychol Methods. 2003;8(3):305.

53. Maxwell SE, Kelley K, Rausch JR. Sample size planning for statistical power and accuracy in parameter estimation. Annu Rev Psychol. 2008; 59(1):537-563.

54. Altman N, Krzywinski M. Points of significance: association, correlation and causation. Nat Meth. 2015;12(10):899-900.

55. Goodwin LD, Leech NL. Understanding correlation: factors that affect the size of r. J Exper Educ. 2006;74(3):249-266.

56. Iacobucci D, Saldanha N, Deng X. A meditation on mediation: evidence that structural equations models perform better than regressions. J Consum Psychol. 2007;17(2):140-154.

57. Edwards JR, Lambert LS. Methods for integrating moderation and mediation: a general analytical framework using moderated path analysis. Psychol Methods. 2007;12(1):1.

58. World Health Organization. Oral Health. Information Sheet. WHO 2012. Available from: http://www.who.int/oral_health/publications/ factsheet/en/.

59. Pitts N, Amaechi B, Niederman R, et al. Global oral health inequalities: dental caries task group - research agenda. Adv Dent Res. 2011;23(2): 211-220.

60. Petersen P, Kandelman D, Arpin S, Ogawa H. Global oral health of older people-call for public health action. Community Dent Health. 2010; 27(4):257-268

61. Skelly AM, Fleming GJ. Perceptions of a dental career among successful applicants for dentistry compared with those of fifth-year dental students. Prim Dent Care. 2002;9(2):41-46.

62. Judd CM, Kenny DA. Estimating the effects of social intervention. New York: Cambridge University Press; 1981.

63. Tanny L, Komabayashi T, Long DL, Yahata Y, Moffat SM, Tane H. The effect of education on oral health students' attitudes in Australia and New Zealand. Eur J Dent. 2016;10(4):491-495. 
64. Ghadimi S, Razeghi S, Khami MR, Zare H. Oral health attitudes and behaviour among medical and dental students in Tehran, Iran. Iraqi $J$ Public Health. 2017;2:30-34.

65. Jaber MF, Khan A, Elmosaad Y, Mustafa MM, Suliman N, Jamaan A. Oral health knowledge, attitude and practices among male Qassim university students. Int J Community Med Public Health. 2017;4(8): 2729-2735.

66. Eitner S, Wichmann M, Schlegel KA, Kollmannsberger JE, Nickenig HJ. Oral health-related quality of life and implant therapy: an evaluation of preoperative, intermediate, and post-treatment assessments of patients and physicians. J Craniomaxillofac Surg. 2012;40(1):20-23.
67. Choi SH, Cha JY, Lee KJ, Yu HS, Hwang CJ. Changes in psychological health, subjective food intake ability and oral health-related quality of life during orthodontic treatment. J Oral Rehabil. 2017;44(11): 860-869.

68. Liu Z, McGrath C, Hagg U. Changes in oral health-related quality of life during fixed orthodontic appliance therapy: an 18-month prospective longitudinal study. Am J Orthod Dentofacial Orthop. 2011;139(2): 214-219.

\section{Publish your work in this journal}

Patient Preference and Adherence is an international, peer-reviewed, open access journal that focuses on the growing importance of patient preference and adherence throughout the therapeutic continuum. Patient satisfaction, acceptability, quality of life, compliance, persistence and their role in developing new therapeutic modalities and compounds to optimize clinical outcomes for existing disease states are major areas of interest for the journal. This journal has been accepted for indexing on PubMed Central. The manuscript management system is completely online and includes a very quick and fair peer-review system, which is all easy to use. Visit http://www. dovepress.com/testimonials.php to read real quotes from published authors.

\footnotetext{
Submit your manuscript here: http://www.dovepress.com/patient-preference-and-adherence-journal
} 